\title{
An Area-Efficient On-Chip Temperature Sensor With Nonlinearity Compensation Using Injection-Locked Oscillator (ILO)
}

\author{
Wongyu Shin, Seungwook Paek, and Lee-Sup Kim \\ Department of Electrical Engineering \\ KAIST \\ Daejeon, Republic of Korea \\ Email: \{wgshin, swpaek\}@mvlsi.kaist.ac.kr, leesup@kaist.ac.kr
}

\begin{abstract}
This paper describes CMOS time-domain temperature sensors. A principle of this type of sensors is CMOS inverter's time-delay variation with temperature. The variation, however, has nonlinearity which is a fundamental error source. Therefore, we propose a new temperature sensor that improves linearity using an injection-locked oscillator (ILO). Since the ILO has the opposite curvature of an inverter delay line in temperature domain, nonlinear error induced by the CMOS inverters can be eliminated. Integral nonlinearity (INL) error is reduced from 3.6 LSB to 0.56 LSB (84\% reduction), resulting in under 1-bit error by nonlinearity. The proposed design has $-0.19^{\circ} \mathrm{C} \sim 0.2^{\circ} \mathrm{C}$ inaccuracy which is only quantization error over $0^{\circ} \mathrm{C} \sim 100^{\circ} \mathrm{C}$. The power consumption is $2.88 \mathrm{~mW}$ including an 8 -bit temperatureto-digital code conversion block at the sampling rate of $15.6 \mathrm{M}$ samples/s.
\end{abstract}

\section{INTRODUCTION}

Many digital systems require on-chip temperature sensors for their own purposes in various fields that need VLSI implementations. There are many examples of usage of on-chip temperature sensors: 1) microprocessor thermal monitoring for dynamic thermal management (DTM) [1]; 2) mobile DRAM self-refresh control [2];3) medical devices to monitor human body conditions [3]; 4) temperature management for backlight systems and power electronics [4].

It is expected that the demand of on-chip temperature sensors will increase continuously with the trend of low-power and system integration. However, conventional on-chip temperature sensors have a limitation due to bipolar transistors (BJT) and analog-to-digital converters (ADC) which are main components of the conventional structure as in Fig. 1. According to [4], BJT-based temperature sensors should be replaced by CMOS-based temperature sensors for full integration into modern VLSI chips. ADC design is getting more difficult with the supply voltage scaling for low-power systems [5]. To overcome these obstacles, CMOS time-domain temperature sensors have been proposed [4], the basic structure of which is depicted in Fig. 2. Temperature dependent delay line (TDDL), consisted of CMOS inverters, makes time-delay induced by thermal variation, and the time-delay is measured by time-to-digital converters (TDC)

CMOS time-domain temperature sensors have advantages in the aspect of system integration and power consumption

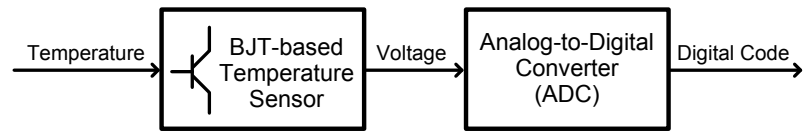

Figure 1. Conventional structure of BJT-based temperature sensor

when considering digital code conversion blocks together, which should be taken into account because the final user of temperature information is digital logic blocks. The area reduction by sharing with clocking systems can also be expected [6]. However, the intrinsic nonlinearity of CMOS inverters, as in Fig. 2, makes fundamental error source, and decreases accuracy significantly.

There is a previous work that solves this problem. Successive approximation register (SAR) control logic is used for nonlinearity compensation [4]. This method is brilliant, but the large area overhead makes designers hesitate to adopt it. In this paper, therefore, we propose a new temperature sensor that improves linearity without area overhead using an injectionlocked oscillator (ILO). A new temperature-to-digital converting scheme is also proposed for optimization into digital VLSI systems.

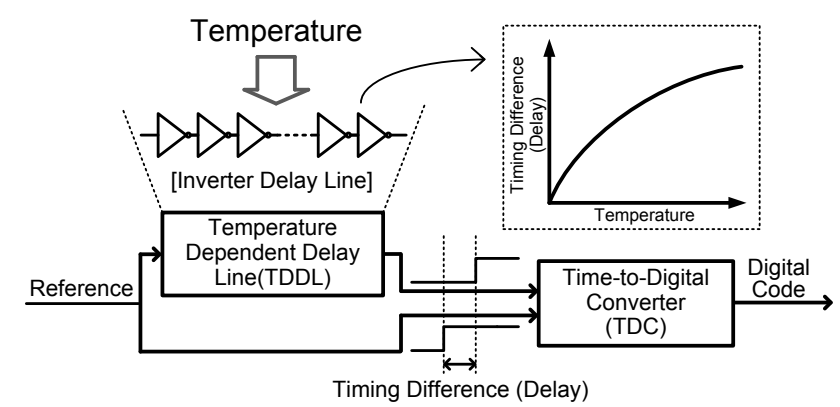

Figure 2. A basic structure of CMOS time-domain temperature sensors and the delay characteristic with temperature for inverter delay lines.

\section{NONLINEARITY COMPENSATION}

A key concept of the proposed design is introduced in this section. It is shown that an ILO can compensate nonlinearity of an inverter delay line. To our knowledge, this is the first work of the analysis of the ILO in temperature domain and using it for temperature sensors. 


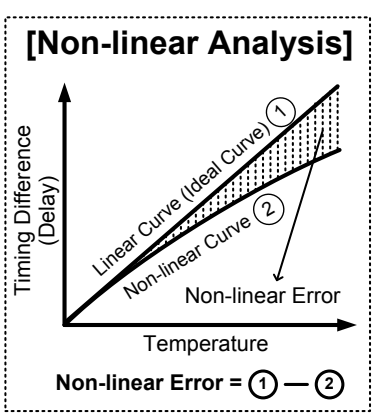

(a)

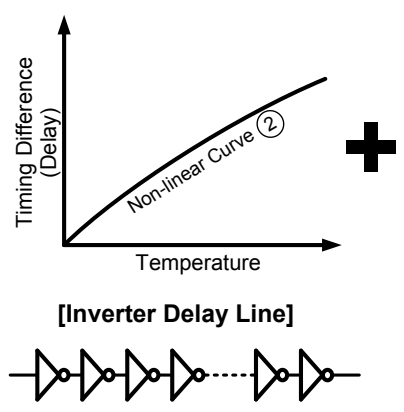

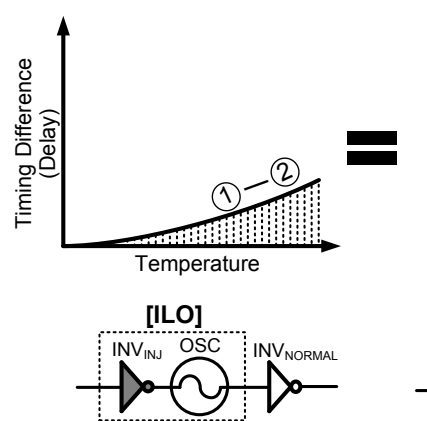

(b)

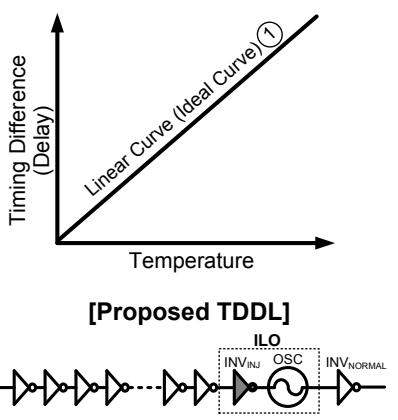

Figure 3. (a) Nonlinear analysis of inverter delay line (b) Nonlinearity compensation by ILO

\section{A. Injection Locked Oscillator (ILO)}

The main idea of this work starts from the characteristic of the ILO which consists of an injection inverter and a CMOS ring-oscillator. The basic feature of the ILO is that the output frequency is locked by an input frequency while output phase is changed according to the free-running frequency of the oscillator and injection strength $(K)$ [7]. Since only the time-delay (phase) should be changed without frequency variation for tuning the delay response of the inverter delay line, the ILO is the most appropriate circuit. In addition, various kinds of delay responses that we need can be shaped by modulation of injection strength $(K)$. We also make the delay response of the ILO by modulating injection strength $(K)$, which is explained in the later section.

\section{B. A key concept of nonlinearity compensation}

Fig. 3 illustrates a key concept of nonlinearity compensation by the ILO. In Fig. 3 (a), the linear curve (ideal curve) is positioned above the nonlinear curve (the delay response of the inverter delay line in temperature domain) to make nonlinear error (1)-(2) positive over the target range. The ILO generates this nonlinear positive error and modifies the delay response of the inverter delay line, resulting in the linear curve as described in Fig. 3 (b). In other words, since the delay response of the ILO has the opposite curvature of the inverter delay line, the nonlinearity of them can be canceled by each other.

While their curvature sign is different, both curves increase with temperature; the second derivative sign is different while the first derivative sign is same. It means that the ILO also makes a contribution for making temperature dependent delay, not carving the delay curve of the inverter delay line (we added delay curve of the ILO to the inverter delay line). From this reason, it is reasonable to regard that the ILO has no area overhead. It is a temperature dependent delay line (TDDL) like the inverter delay line. The only difference is the sign of curvature.

\section{Curvature shaping of ILO in temperature domain}

Our purpose is to make the curve which has positive curvature for nonlinearity compensation. First, we draw the delay of the ILO in time domain, and shape the curve which has positive curvature in temperature domain by modulating injection strength $(K)$.

Fig. 4 (a) is a schematic of the ILO and Fig. 4 (b) is a process that makes the final delay of the ILO. D1/D3 is a timedelay of $\mathrm{INV}_{\mathrm{INJ}} / \mathrm{INV}_{\mathrm{NORMAL}}$, and $D 2$ is a time-delay of multiple
$\mathrm{INV}_{\mathrm{OSC}} \mathrm{S}$ that compose the ring-oscillator. An injection signal is fed into the circuit through path $<1>$ and an oscillating signal is circulated through path $<2>$. On the OSC_Mid node, two signals from path $<1>$ and path $<2>$ are mixed, and a delay is calculated by phase interpolation of these two signals. This is represented as follows:

$$
D_{n+1}=\left(D_{n}+D_{2}\right) \cdot \frac{A}{A+B}
$$

The created signal is circulated again through path $\langle 2\rangle$, and a new delay is calculated again by phase interpolation with the injecting signal from path $<1>$. The consecutive phase interpolations result in the delay as follows:

$$
D_{n}=\sum_{r=1}^{n}\left(\frac{A}{A+B}\right)^{r} \cdot D_{2}
$$

The final delay is derived as follows:

$$
\begin{aligned}
& D_{\infty}=\lim _{n \rightarrow \infty} \sum_{r=1}^{n}\left(\frac{A}{A+B}\right)^{r} D_{2}=\frac{\left(\frac{A}{A+B}\right) D_{2}}{1-\left(\frac{A}{A+B}\right)}=\frac{D_{2}}{K} \\
& \text { where K(Injection strength) }=I_{I N J} / I_{O S C}=B / A
\end{aligned}
$$

The final signal is fed out through $\mathrm{INV}_{\text {NORMAL }}$, and $D_{\text {FINAL }}$ is determined as follows:

$$
D_{\text {FINAL }}=D_{1}+\frac{D_{2}}{K}+D_{3}
$$

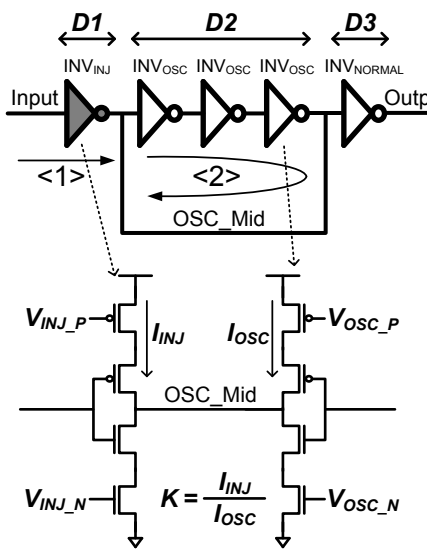

(a)

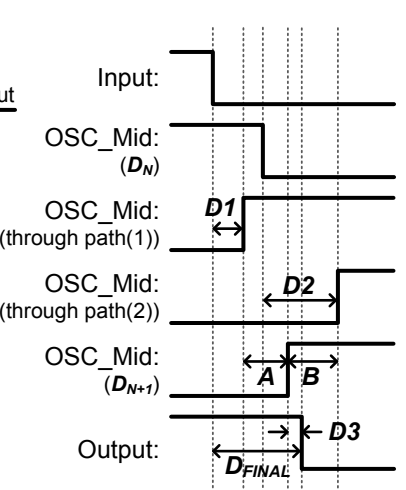

$A: B=I_{\text {osc }}: I_{\text {INJ }}$

(b)
Figure 4. (a) Schematic of ILO (b) A process that makes the final delay 
Then, we must make $D_{\text {FINAL }}$ to have positive curvature in temperature domain. Fig. 5 describes that positive curvature can be created by modulating injection strength $(K)$. Since $D 1$ and $D 3$ are much smaller than $D 2$, temperature dependent delay of $D 1$ and $D 3$ can be regarded as negligible. For $D 2 / K$ to have positive curvature, $K$ should be decreased because $D 2$ increases with temperature as in Fig. 5 (a). In the same manner, for $K$ to decrease, $I_{I N J}$ should be decreased faster than $I_{O S C}$ with temperature as in Fig. 5 (b). This is represented as follows:

$$
\left|\frac{d I_{I N J}}{d T}\right|>\left|\frac{d I_{O S C}}{d T}\right|
$$

Finally, to make the condition as (5), we change $V_{I N J N}$ and $V_{O S C}$ because current response is different according to bias voltage. $V_{I N J_{-} P}$ and $V_{O_{S C} P}$ are generated by current mirror circuits. Consequently, the delay response in temperature domain can be shaped by modulating $V_{I N J_{-} N}$ and $V_{O S C}{ }_{N}$. In the proposed design, the optimal $V_{I N J N}$ and $V_{O S C}{ }_{N}$ are $850 \mathrm{mV}$ and $760 \mathrm{mV}$ to shape the temperature dependent delay that has positive curvature. Fig. 5 (c) shows the simulation results.
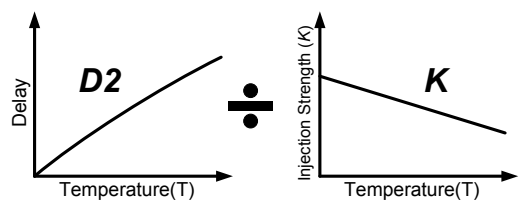

(a)
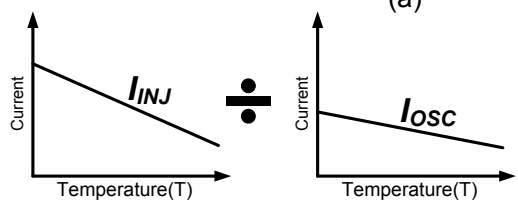

(b)


(c)

Figure 5. Positive curvature shaping in temperature domain (a) $D 2 / K$ shaping (b) Injection strength $(K)$ shaping (c) simulation results of $K$ shaping

\section{TEMPERATURE-TO-DIGITAL COVERTER}

Since the advantage of on-chip temperature sensors exists in integration with other VLSI systems, it is important to consider the temperature-to-digital converting method in the environment where the temperature sensors are equipped. Therefore, in this section, temperature-to-digital converting scheme that is optimized into digital VLSI systems is introduced.

\section{A. Overall Architecture}

Fig. 6 (a) shows an overall architecture of the proposed temperature sensor. A delay-locked loop (DLL) is used to make temperature-independent delay which is a reference for comparison with temperature dependent delay. The DLL also provides multi-phases for digital code converting. Using DLL has the advantage of area reduction because it can be shared by DLLs which are already equipped in many digital systems [6].

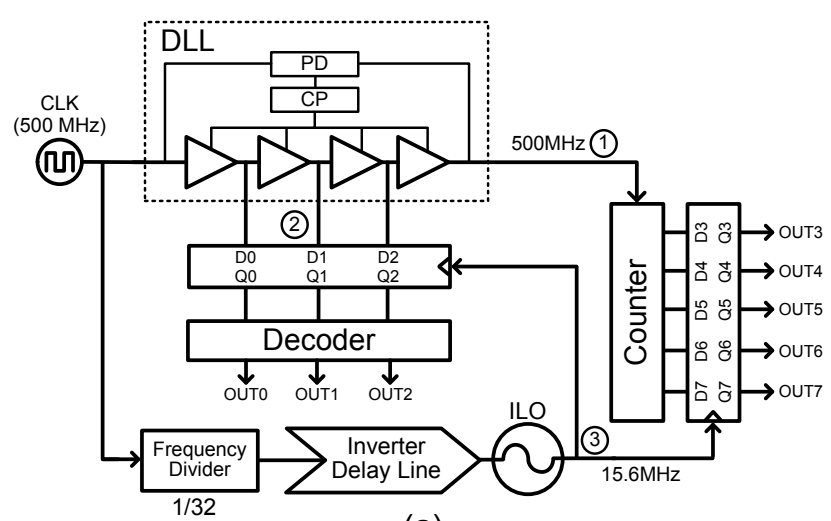

(a)

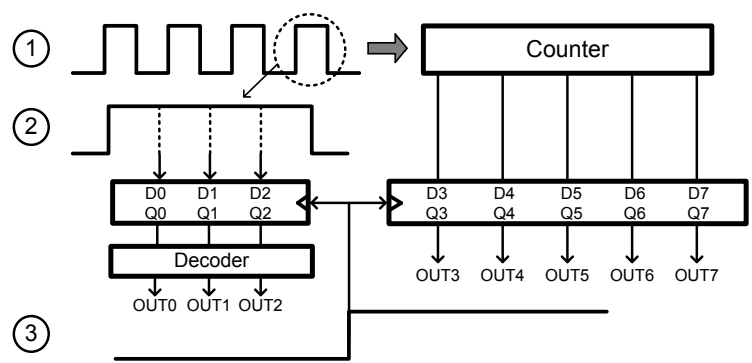

(b)

Figure 6. (a) Overall architecture of the proposed design (b) Temperature-todigital code converting process

The temperature dependent delay line (TDDL) consists of the inverter delay line and ILO as explained before. The counter and decoder are equipped to generate digital code.

\section{B. Temperature-to-digital code converting scheme}

Fig. 6 (b) describes a temperature-to-digital converting process. The counter and decoder generate digital codes using the reference clock and multi-phases made by the DLL, and the signal after TDDL samples the digital codes. Since digital codes are from temperature independent signal after DLL, a digital code sampled by the signal after TDDL becomes a temperature value. Input clock is decided as $500 \mathrm{MHz}$ to share DLL of the system that use this temperature sensor. A lot of digital systems operate at around $500 \mathrm{MHz}$, and DLLs equipped in the system are also designed at around $500 \mathrm{MHz}$.

Fine measurement is performed by the decoder and coarse measurement is performed by the counter. This gives flexibility to digital logic blocks which use temperature information. If they need low resolution, they can turn the decoder off to save power. Some applications need different resolution depending on situation.

\section{SimUlation RESUlts}

The proposed temperature sensor is designed and simulated using Cadence Spectre with 130nm CMOS technology under $1.2 \mathrm{~V}$ supply voltage. The measurement range is from $0{ }^{\circ} \mathrm{C}$ to $100{ }^{\circ} \mathrm{C}$ with $0.39^{\circ} \mathrm{C} / \mathrm{LSB}$ resolution. This specification satisfies almost all requirements of VLSI systems. Input reference frequency is $500 \mathrm{MHz}$ and it is divided by 32 for the input of temperature dependent delay line (TDDL). The sampling rate 
is $15.6 \mathrm{M}$ samples/s. It is enough for tracking temperature variation. Systems that use this temperature sensor can have flexibility to control sampling rate depending on situation. After two-point calibration, the simulation is performed.

Fig. 7 shows the improvement of linearity by the ILO. When the inverter delay line and the proposed TDDL (inverter delay line + ILO) have the same delay at $100^{\circ} \mathrm{C}$, the inverter delay line has 900 ps nonlinear error while the proposed TDDL only has 140 ps nonlinear error. 760 ps is reduced $(84 \%$ reduction). The corresponding integral nonlinearity (INL) error is lowered from 3.6 LSB to $0.56 \mathrm{LSB}$, resulting in under 1-bit nonlinearity error.

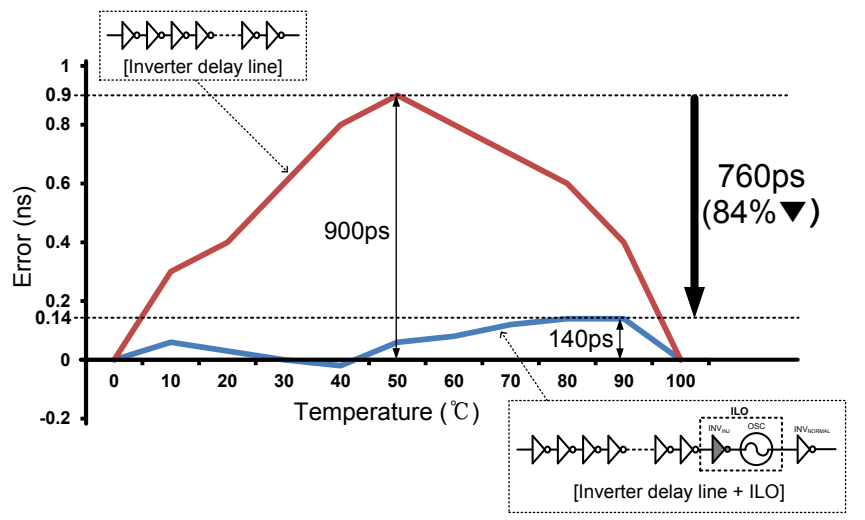

Figure 7. Simulation result of nonlinear error (nonlinearity comparison between inverter delay line and proposed TDDL)

Fig. 8 is a layout of the proposed temperature sensor. The area is much smaller than the previous work that performs nonlinearity compensation [4].

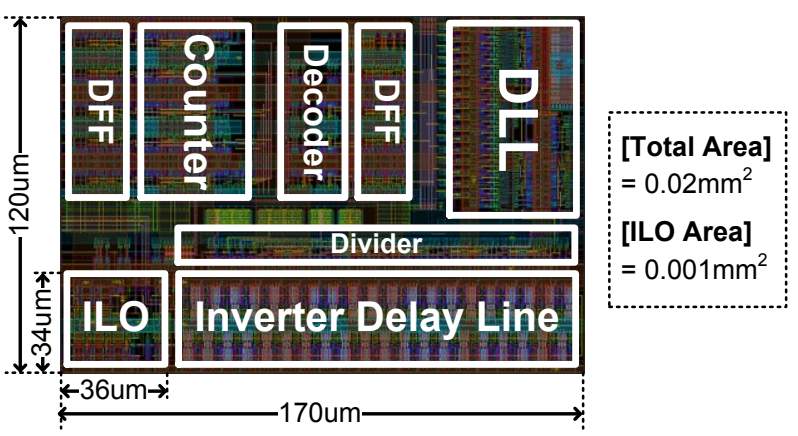

Figure 8. Layout of the proposed temperature sensor

Table I shows the comparison with the previous works that use CMOS inverter delay line. It is verified that this work performs nonlinearity compensation with much less overhead compared with the previous work.

\section{CONCLUSION}

This paper proposes a new temperature sensor focusing on improving linearity without area overhead. We found the method of shaping delay response in temperature domain using an ILO, and make the delay that has positive curvature to cancel the error from negative curvature of an inverter delay
TABLE I. COMPARISON WITH OTHER WORKS

\begin{tabular}{|c|c|c|c|}
\hline Parameter & {$[8]$} & {$[4]$} & This work* \\
\hline Technology $(\mathrm{um})$ & 0.13 & 0.35 & 0.13 \\
\hline Area $\left(\mathrm{mm}^{2}\right)$ & 0.16 & 0.6 & 0.02 \\
\hline Error $\left({ }^{\circ} \mathrm{C}\right)$ & $-1.8 \sim 2.3$ & $-0.25 \sim 0.35$ & $-0.19 \sim 0.2$ \\
\hline Resolution $\left({ }^{\circ} \mathrm{C} / \mathrm{LSB}\right)$ & 0.66 & 0.0918 & 0.39 \\
\hline $\begin{array}{c}\text { Temperature } \\
\text { Range }\left({ }^{\circ} \mathrm{C}\right)\end{array}$ & $0 \sim 100$ & $0 \sim 90$ & $0 \sim 100$ \\
\hline Power $(\mathrm{mW}) * *$ & 1.2 & 35 & 2.88 \\
\hline $\begin{array}{c}\text { Nonlinearity } \\
\text { Compensation }\end{array}$ & No & Yes & Yes \\
\hline
\end{tabular}

simulation result

**when operating at the full conversion rate that each design has.

line. INL error is reduced by $84 \%$, resulting in under 1 -bit nonlinearity error. Temperature-to-digital converting scheme is also proposed which is optimized for digital VLSI systems. The area of this proposed temperature sensor is $0.02 \mathrm{~mm}^{2}$ which is much smaller than the previous works.

\section{ACKNOWLEDGMENT}

The chip fabrication was provided by MPW of IDEC. This research was supported by Basic Science Research Program through the NRF of Korea funded by MEST (2013004051).

\section{REFERENCES}

[1] Seungwook Paek, Wongyu Shin, Jaeyoung Lee, Hyo-Eun Kim, JunSeok Park and Lee-Sup Kim, "All-Digital Hybrid Temperature Sensor Network for Dense Thermal Monitoring," IEEE International SolidState Circuits Conference Digest of Technical Papers, pp.260-261, Feb. 2013.

[2] Chan-Kyung Kim, Bai-Sun Kong, Chil-Gee Lee, and Young-Hyun Jun, "CMOS Temperature Sensor with Ring Oscillator for Mobile DRAM Self-refresh Control," IEEE International Symposium on Circuits and Systems, pp.3094-3097, May 2008.

[3] Oh-Yong Jung, Seungjin Kim, Sok-Kyun Han, and Sang-Gug Lee, "A Low Power Low Inaccuracy Linearity-Compensated Temperature Sensor for Attachable Medical Devices," IEEE International Symposium on Circuits and Systems, pp.1087-1090, May 2013.

[4] Poki Chen, Chun-Chi Chen, Yu-Han Peng, Kai-Ming Wang, and YuShin Wang, "A Time-Domain SAR Smart Temperature Sensor With Curvature Compensation and a $3 \sigma$ Inaccuracy of $-0.4^{\circ} \mathrm{C} \sim+0.6^{\circ} \mathrm{C}$ Over a $0{ }^{\circ} \mathrm{C}$ to $90^{\circ} \mathrm{C}$ Range," IEEE Journal of Solid-State Circuits, pp.600-609, March 2010.

[5] Y. William Li and Hasnain Lakdawala, "Smart Integrated Temperature Sensor - Mixed-Signal Circuits and Systems in 32-nm and Beyond," IEEE Custom Integrated Circuits Conference, pp.1-8, Sept. 2011.

[6] Seungwook Paek, Jiehwan Oh, Sang-Hye Chung, and Lee-Sup Kim, "Area-Efficient Dynamic Thermal Management Unit using MDLL with Shared DLL Scheme for Many-Core Processors," IEEE International Symposium on Circuits and Systems, pp.1664-1667, May 2011.

[7] Kangmin $\mathrm{Hu}$, Tao Jiang, Jingguang Wang, Frank O'Mahony, and Patrick Yin Chiang, "A $0.6 \mathrm{~mW} / \mathrm{Gb} / \mathrm{s}, 6.4-7.2 \mathrm{~Gb} / \mathrm{s}$ Serial Link Receiver Using Local Injection-Locked Ring Oscillators in $90 \mathrm{~nm}$ CMOS," IEEE Journal of Solid-State Circuits, pp.899-908, April 2010.

[8] Kyoungho Woo, Scott Meninger, Thucydides Xanthopoulos, Ethan Crain, and Donhee Ham, "Dual-DLL-based CMOS All-Digital Temperature Sensor for Microprocessor Thermal Monitoring," IEEE International Solid-State Circuits Conference Digest of Technical Papers, pp.68-69, Feb. 2009. 